\title{
Faxback Response
}

Name:

Address:

City:

State:

ZIP:

E-mail:

Specialty:

Signature:

Date:

Fax this form to 212.328.0600. Or mail it to:

CNS SPECTRUMS

MBL Communications, Inc.

\section{Hudson Street, 7th Floor}

New York, NY 10013

Your comments are important to us. This form provides you with the opportunity to express your opinions. Our goal is to make CNS Spectrums your source for practical and clinical neuropsychiatric information. By filling out this FaxBack form, you enable us to incorporate your views about our editorial content in future issues. Please fill out this form in its entirety. Thank you.

1. On a scale of 1 to 5 ( $1=$ Poor, $5=$ Excellent), please indicate your level of interest and/or satisfaction with the editorial content in this issue.

\section{Cover Topic: Late-life Depression and Dementia}

$$
\begin{array}{lllll}
1 & 2 & 3 & 4 & 5
\end{array}
$$

\section{Departments}

\section{CNS News}

$\begin{array}{lllll}1 & 2 & 3 & 4 & 5\end{array}$

CME

$$
\begin{array}{lllll}
1 & 2 & 3 & 4 & 5
\end{array}
$$

\section{Teaching Monograph Supplement}

$$
\begin{array}{lllll}
1 & 2 & 3 & 4 & 5
\end{array}
$$

2. Which areas of neuropsychiatry would you like us to cover in the future? 


\section{SUBSGRIPTION INFORMATION}

$\square$ YES! I want to continue my free subscription to CNS SPECTRUMS

Name:

Address:

City:

State:

ZIP:

E-mail:

Specialty:

Signature:

Date:

*Please complete all fields

Fax this form to 212.328.0600. Or mail it to:

CNS SPECTRUMS

MBL Communications, Inc.

333 Hudson Street, 7th Floor

New York, NY 10013 


\section{Guide TO DSM-IV AND IGD-10 GODES}

Dementia of the Alzheimer Type, With Early Onset With Depressed Mood

DSM-IV

ICD-10

Specify if: With Behavioral Disturbance

290.13

F00.03

Specify if: With Behavioral Disturbance

290.21

Delirium Due to: Indicate General Medical Condition

293.0

Psychotic Disorder Due to: Indicate General Medical Condition With Delusions

With Hallucinations

Mood Disorder Due to: Indicate General Medical Condition

Anxiety Disorder Due to: Indicate General Medical Condition

Amnestic Disorder Due to: Indicate General Medical Condition

Dementia NOS

Amnestic Disorder NOS

Schizophrenia

Schizophrenia-Disorganized Type

Schizophrenia-Catatonic Type

Schizophrenia-Paranoid Type

Schizophrenia-Residual Type

Schizoaffective Disorder

Schizophrenia-Undifferentiated Type

Major Depressive Disorder

Bipolar I Disorder

Bipolar Disorder NOS

Bipolar II Disorder

Mood Disorder NOS

Psychotic Disorder NOS

Autistic Disorder

Asperger's Disorder

Pervasive Developmental Disorder NOS

Anxiety Disorder NOS

Panic Disorder Without Agoraphobia

Generalized Anxiety Disorder

Dissociative Identity Disorder

Dissociative Disorder NOS

Factitious Disorder NOS

Panic Disorder With Agoraphobia

Agoraphobia Without History of Panic Disorder

Social Phobia

Specific Phobia

Obsessive-Compulsive Disorder

Dysthymic Disorde

Depersonalization Disorder

Body Dysmorphic Disorder

Somatization Disorder

Somatoform Disorder NOS

Cyclothymic Disorder

Alcohol Dependence

Cocaine Dependence

Cannabis Dependence

Amphetamine Dependence

Alcohol Abuse

Cannabis Abuse

Cocaine Abuse

Amphetamine Abuse

Stuttering

Anorexia Nervosa

Tic Disorder NOS

Tourette Disorde

Primary Insomnia

Primary Hypersomnia

Sleepwalking Disorder

Dyssomnia NOS

Nightmare Disorder

Parasomnia NOS

Eating Disorder NOS

Bulimia Nervosa

Feeding Disorders of Infancy or Early Childhood

Communication Disorder NOS

Posttraumatic Stress Disorder

Depressive Disorder NOS

Impulse-Control Disorder NOS

Pathological Gambling

Pyromania

Kleptomania

Trichotillomania

Disruptive Behavior Disorder NOS

Attention-Deficit/Hyperactivity Disorder, Combined Type

Attention-Deficit/Hyperactivity Disorder NOS

Learning Disorder NOS

Developmental Coordination Disorder

Narcolepsy

Sleep Disorder Due to: Indicate General Medical Condition

Delirium NOS

F00.13

F05.0

F06.2

F06.0

F06

F06.4

FO2.8

$\mathrm{FO} 3$

R41.3

F20

F20.1

F20.2

F20.0

F20.5

F25

F20.3

F32

F30

F39

F31.8

F39

F29

F84

F84.5

F84.9

F41.9

F41

F41.1

F44.81

F44.9

F68.1

F40.01

F40

F40.1

F40.2

F42.8

F34.1

F48.1

F45.2

F45.

F45.9

F34

F10.2

F14.2

F12.2

F15.2

F10.1

F12.1

F14.1

F15.1

F98.5

F50

F95.9

F95.2

F51.0

F51.1

F51.3

F51.9

F51.5

F51.8

F50.9

F50.2

F98.2

F80.9

F43.1

F32.9

F63.9

F63.0

F63.1

F63.2

F63.3

F91.9

F90

F90.9

F81.9

F82

G47.4

G47

F05.9 
BRIEF SUMMARY. See package insert for full prescribing information. CONTRAINDICATIONS: Hypersensitivity to venlafaxine hydrochloride or to any excipients in the formulation. Concomitant use in patients taking monoamine
oxidase inhibitors (MADIs) is contraindicated. WARNINGS: Potential for Interaction with Monoamine Oxidase Inhibitors-Adverse reactions, some serious, have been reported in patients who were recently discontinued from an $\mathrm{MAOI}$ and started on venlafaxine, or who recentty had venlataxine therapy discontinued prior to initiation of an MAOl. These reactions included tremor, myoctonus, diaphoresis, nausea, vomiting, flushing dizziness, hyperthermia with features resembling neuroleptic malignant syndrome, seizures, and death. is recommended that Effexor XR not be used in combination with an MAOl, or within at least 14 days of discontinuing treatment with an MAOI. Based on the half-life of venlafaxine, at least 7 days should be allowed after stopping venlafaxine before starting an MAOI. Sustained Hypertension-Venlafaxine is associated with sustained increases in blood pressure (BP) in some patients. Experience with immediate release venlafaxine showe that sustained hypertension was dose related. It is recommended that patients receiving Effexor XR have regular monitoring of $\mathrm{BP}$. For patients who experience a sustained increase in $\mathrm{BP}$ either dose reduction or discontinuation should be considered. PRECAUTIONS: General-insomnia and Nervousness: Treatment-emergent insomnia an nervousness have been reported. Insomnia and nervousness each led to drug discontinuation in $0.9 \%$ of the patients
in Phase 3 depression studies. In Phase 3 Generalized Anxiety Disorder (GAD) trials, insomnia and nervousness led to drug discontinuation in 3\% and $2 \%$, respectively, of patients. Changes in Appetite Weight: Treatment-emerge anorexia has been reported. A loss of $5 \%$ or more of body weight occurred in $7 \%$ of patients in placebo-controlle depression trials. A loss of $7 \%$ or more of body weight occurred in $3 \%$ of patients in placebo-controlled GAD trials. The safety and efficacy of venlafaxine therapy in combination with weight loss agents, including phentermine, have not been established. Coadmint hypomania has occurred during short-term depression studies. Effexor XR should be used cautiously in patients with a history of mania. Hyponatremia: Hyponatremia and/or the syndrome of inappropriate antidiuretic hormon secretion (SIADH) may occur with venlafaxine. This should be taken into consideration in patients who are, for example secretion (SIADH) may occur with venlafaxine. This should be taken into consideration in patients who are, for example
volume-depleted, elderly, or taking diuretics. Mydriasis: Mydriasis has been reported; therefore patients with raised volume-depleted, elderly, or taking diuretics. Mydriasis: Mydriasis has been reported; therefore patients with raise intraocular pressure or at risk of acute narrow-angle glaucoma should be monitored. Seizures: In all premarketing depression trials with Effexor, seizures were reported in $0.3 \%$ of venlafaxine-treated patients. Use Effexor XR cautiously have been reports of abnormal bleeding (most commonly ecchymosis). Suicide: The possibility of a suicide attempt is inherent in depression and may persist until significant remission occurs. Closely supervise high-risk patien during initial dnig therapy. Prescriptions for Effexor XR should be written for the smallest quantity of capsules consistent with good patient management to reduce the risk of overdose. The same precautions should be observed when treating patients with GAD. Use in Patients With Concomitant lliness: Use Effexor XR cautiously in patients with disease with recent history of Ml or unstable heart disease. In short-term depression studies electrocardiographic changes in corrected QT interval (QTc) showed a mean increase of $4.7 \mathrm{msec}$, and the mean change from baseline heart rate was 4 beats per minute. In GAD studies, mean changes in QTC did not differ significantly from placebo and the mean 4 beats per minute. In GAD studies, mean changes in QTC did not differ significantly from placebo and the mean (mean dose $>300 \mathrm{mg} /$ day) patients had a mean increase in heart rate of 8.5 beats per minute. Caution should be exercised in patients whose underlying medical conditions might be compromised by increases in heart rate (e.g. patients with hyperthyroidism, heart failure, or recent Mi). In patients with renal impairment or cirrhosis of the liver, the clearances of venlafaxine and its active metabolites were decreased, thus prolonging the elimination half-lives. A lowe dose may be necessary; use with caution in such patients. Information for Patients-Caution patients abo operating hazardous machinery, including automobiles, until they are reasonably sure that venlataxine does not adversely affect their abilities. Tell patients to avoid alcohol while taking Effexor XR and to notify their physician 1) they become pregnant or intend to become pregnant during therapy, or if they are nursing; 2) about other prescriptio or over-the-counter drugs, including herbal preparations, they are taking or plan to take; 3) if they develop a rash, Interactions-Alcohol: A single dose of ethanol had no effect on the pharmacokinetics of venlafaxine or 0 -desmethylvenlafaxine (ODY when venlafaxine was administered and venlafaxine did not exaggerate the psychomotor and tered and venlafaxine did not exaggerate the psychomotor and
psychometric effects induced by ethanol. Cimetidine: Use with caution when administering venlataxine with cimetidine to patients with pre-existing hypertension or hepatic dystunction and the elderly. Diazepam: A single dose of diazepam did not appear to affect the pharmacokinetics of either venlafaxine or
ODV Venlafaxine did not have any effect on the pharmacokinetics of diazepam or its active metabolite, desmethyldiazepam, or affect the psychomotor and psychometric effects induce by diazepam. Haloperidol: Venlafaxine decreased total oral-dose clearance of haloperidol which resulted in a $70 \%$ increase in haloperidol AUC. The haloperidol $\mathrm{C}_{\text {max }}$ increased $88 \%$ when coadministered with venlafaxine, but pharmacokinetics of either venlafaxine or ODV. Venlafaxine had no effect on the pharmacokinetics of lithium. Drugs inhibiting Cytochrome P450206 Metabolism: Venlafaxine is metabolized to its active metabolite, ODV, via crochrome $\mathrm{P} 450206$. Drugs inhibiting this isoenzyme have the potential to increase plasma concentrations of cyrochrome P450206. Drugs inhibiting this isoenzyme have the potential to increase plasma concentrations venlafaxine and decrease concentrations of ODV. Since the composite plasma levels of venlafaxine and OOV are
essentially unchanged in CYP2D6 poor metabolizers, no dosage adjustment is required when venlafaxine is essentially unchanged in CYP2D6 poor metabolizers, no dosage adjustment is required when venlafaxine
coadministered with a CYP2D6 inhibitor. The concomitant use of venlafaxine with a drug treatment(s) that potentially inhibits both CYP2D6 and CYP3A4, the primary metabolizing enzymes for venlafaxine, has not been studied. Caution is advised should a patient's therapy include venlafaxine and any agent(s) that produce simultaneous inhibition of these
two enzyme systems. Dngs Metabolized by Cytochrome P450 Isoenzymes: Studies indicate that venlafaxine is relatively weak inhibitor of CYP2D6. Venlafaxine did not inhibit CYP1A2 and CYP3A4, CYP2C9 (in vitro), or CYP2C19. Imipramine: Venlataxine did not affect the pharmacokinetics of imipramine and 2-OH-imipramine. However desipramine $\mathrm{AUC}, \mathrm{C}_{\max }$ and $\mathrm{C}_{\min }$ increased by about $35 \%$ in the presence of venlafaxine. The 2-OH-desipramine AUC' increased by $2.5-4.5$ fold. Imipramine did not affect the pharmacokinetics of venlafaxine and OOV. Risperidone Venlafaxine slightly inhibited the CYP2D6-mediated metabolism of risperidone to its active metabolite, 9-hydroxyrisperidone, resulting in an approximate $32 \%$ increase in risperidone AUC. Venlafaxine coadministration did not significantly alter the pharmacokinetic profile of the total active moiety (risperidone plus 9-hydroxyrisperidone) Indinavir: In a study of 9 healthy volunteers, venlataxine resulted in a $28 \%$ decrease in the AUC of a single dose indinavir and a $36 \%$ decrease in indinavir $C_{\text {max }}$. Indinavir did not affect the pharmacokinetics of venlafaxine and 00 MAOls: See "Contraindications" and "Warnings." cuS-Active Drus: Caution is advised if the concomitant administration of venlafaxine and CNS-active drugs is required. Carcinogenesis, Mutagenesis, Impairment of FertilityCarcinogenesis: There was no increase in tumors in mice and rats given up to 1.7 times the maximum recommended human dose (MRHD) on a $\mathrm{mg} / \mathrm{m}^{2}$ basis. Mutagenesis: Venlafaxine and ODV were not mutagenic in the Ames reverse mutation assay in Salmonella bacteria or the Chinese hamster ovary/HGPRT mammalian cel torward gene mutation assay. Venlafaxine was not clastogenic in several assays. ODV elicited a clastogenic response in the in vivo chromosomal aberration assay in rat bone marrow. Impaiment of fertifity: No effects on reproduction or fertility in rats were noted at oral doses of up to 2 times the MRHD on a $\mathrm{mg} / \mathrm{m}^{2}$ basis. Pregnancy-Teratogenic $\left(\mathrm{mg} / \mathrm{m}^{2}\right.$ basis) revealed no malformations in offspring. However, in rats given 2.5 times the MRHD, there was decrease in pup weight, an increase in sti:lbom pups, and an increase in pup deatts during the first 5 days of lactation when dosing began during pregnancy and continued until weaning. There are no adequate and well-controlled when dosing began during pregnancy and continued until weaning. There are no adequate and well-controlled studies in pregnant women; use Effexor XR during pregnancy only if clearly needed. Nonteratogenic Effects. If
venlafexine is used until or shortly before birth, discontinuation effects in the newborn should be considered. Labor Delivery, Nursing -The effect on labor and delivery in humans is unknown. Venlafaxine and ODV have been reported to be excreted in human milk. Because of the potential for serious adverse reactions in nursing infants from Effexor $X R$, a decision should be made whether to discontinue nursing or to discontinue the drug, taking into account the importance of the drug to the mother. Pediatric Use - Safety and effectiveness in pediatric patients have not been established. Geriatric Use-Approximately $4 \%$ and $6 \%$ of Effexor XR-treated patients in placebo-controlled premarketing depression and GAD trials, respectively, were 65 years of age or over. No overall differences in effectiveness o safety were observed between geriatric patients and younger patients. However, greater sensitivity of some olde secretion (SIADH) have been reported, usually in the elderly. ADVERSE REACTIONS: Associated with Discontinuation of Treatment-The most common events leading to discontinuation in depression and GAD trials included: nausea, anorexia, dry mouth, dizziness, insomnia, somnolence, hypertension, diarthea, paresthesia, tremor abnormal (mostyy blurred) vision, abnormal (mostly delayed) ejaculation, asthenia, vomiting nervousness, and sweating abnormal (mostly blurred) vision, abnormal (mostly delayed) ejaculation, asthenia, vomiting, nervousness, and sweating asthenia. Cardiovascular: vasodilatation, hypertension. Digeșive: nausea, constipation, anorexia, vomiting, flatulence Metabolic Nutritional: weight loss. Nervous System: dizziness, somnolence, insomnia, dry mouth, nervousness, abnormal wann. Skin: or, depression, hypertonia, paresthesia, libido decreased, agitation. Respiratory Skstem. phany gits,

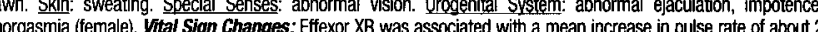

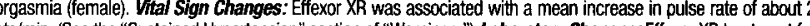

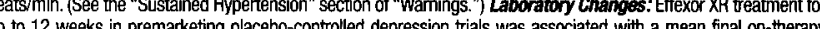
increase in serum cholesterol concentration of approximately $1.5 \mathrm{mg} / \mathrm{dL}$. Effexor XR treatment for up to 8 weeks and up to 6 months in premarketing placebo-controlled GAD trials was associated with mean final on-therapy increases in serum cholesterol concentration of approximately $1.0 \mathrm{mg} / \mathrm{dL}$ and $2.3 \mathrm{mg} / \mathrm{dL}$, respectively. Patients treated with Effexor tablets (the immediate-release form of venlafaxine) for at least 3 months in placebo-controlled 12-month extension trials had a mean final on-therapy increase in total cholesterol of $9.1 \mathrm{mg} / \mathrm{dl}$. This increase was duration dependent over the 12-month study period and tended to be greater with higher doses. An increase in serum cholesterol from baseline by $\geq 50 \mathrm{mg} / \mathrm{dL}$ and to values $>260 \mathrm{mg} / \mathrm{dL}$, at any time after baseline, has been recorded in 8.1\% of patients. ECG Changes: See the "Use in Patients with Concomitant linesses" section of PRECAUTONS. Other Events Observed During the Premarketing Evaluation of Effexor and Effexor $X R-N=5079$. "Frequent" $=$ events
occuring in at least $1 / 100$ patients; "infrequent" $=1 / 100$ to $1 / 1000$ patients; "rare" = fewer than $1 / 1000$ patients. Body occurring in at least $1 / 100$ patients; "infrequent" $=1 / 100$ to $1 / 1000$ patients; "rare"=fewer than $1 / 1000$ patients. Bods as a whole - Frequent: chest pain substemal, chills, fever, neck pain; Infrequent: face edema, intentional injury, appendicitis, bacteremia, carcinoma, cellulitis. Gardiovascular system - Frequent: migraine, postural hypotension, cold feet and/or cold hands), syncope, thrombophlebitis; Rare: aortic aneurysm, arteritis, first-degree atrioventricular block, bigeminy, bradycardia, bundle branch block, capillary fragility, cerebral ischemia, coronary artery disease, congestive heart failure, heart arrest, cardiovascular disorder (mitral valve and circulatory disturbance), mucocutaneous hemonthage, myocardial infarct, pallor. Digestive system - Frequent enuctation, increased appetite; Infequent: bruxism, colitis, dysphagia, tongue edema, esophagitis, gastritis, gastroenteritis, gastrointestinal ulcer, gingivitis, glossitis, rectal hemorthage, hemornoids, melena, oral moniliasis, stomatitis, mouth ulceration; Rare: cheilitis, cholecystitis, cholelithiasis, esophageal spasms, duodenitis, hematemesis, gastrointestinal hemornhage, gum hemornage, hepatitis, ileitis, jaundice, intestinal obstruction, parotitis, proctitis, increased salivation, soft stools, tongue discoloration Endocrine system - Rare: goiter, hyperthyroidism, hypothyroidism, thyroid nodule, thyroiditis. Hemic and lymphatic system - Frequent: ecchymosis; Infrequent: anemia, leukocytosis, leukopenia, lymphadenopathy, thrombocythemia thrombocytopenia; Rare: basophilia, bleeding time increased, cyanosis, eosinophilia, lymphocytosis, multiple myeloma, purpura. Metabolic and nutritional - Frequent: edema, weight gain; Infrequent: alkaline phosphatase increased, dehydration, hypercholesteremia, hyperglycemia, hyperlipemia, hypokalemia, SGOT increased, SGPT increased, thirst; abnormal, hemochromatosis, hypercalcinuria, hyperkalemia, hyperphosphatemia, hyperuricemia, hypocholesteremia, hypoglycemia, hyponatremia, hypophosphatemia, hypoproteinemia, uremia. Musculoskelotal system - Frequent: artholgaical fracture, myopathy, osteoporosis, osteosclerosis, theumatoid arthritis, tendon nupture. Neryous system pathological fracture, myopathy, osteoporosis, osteosclerosis, reumatoid arthritis, tendon npture. Hervous system Infrequent: apatty, ataxia, circumoral paresthesia, CNS stimulation, euphoria, hallucinations, hosility, hyperesthesia hyperkinesia, hypotonia, incoordination, manic reaction, myoclonus, neuralgia, neuropathy, psychosis, seizure, abnormal speech, stupor, twitching; Rare: akathisia, akinesia, alcohol abuse, aphasia, bradykinesia, buccoglossal syndrome,
cerebrovascular accident, loss of consciousness, delusions, dementia, dystonia, facial paralysis, abnomal gait, Guillain-Barre Syndrome, hyperchlorhydria, hypokinesia, impulse control difficulties, libido increased, neuritis, nystagmus, paranoid reaction, paresis, psychotic depression, reflexes decreased, reflexes increased, suicidal ideation, torticollis. Respiratony system - Frequent: cough increased, dyspnea; Infrequent: asthma, chest congestion, epistaxis, hyper-
ventilation, laryngismus, laryngitis, pneumonia, voice alteration; Rare: atelectasis, hemootysis, hypoventilation, hypoxia, larynx edema, pleurisy, pulmonary embolus, sleep apnea. Skin and appendages - Frequent: rash, pruritus; Infrequent: acne, alopecia, brittle nails, contact dermattis, dry skin, eczema, skin hypertophy, maculopapular rash,
Ne

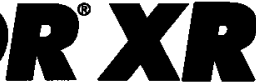

EXTENDED RELEASE CAPSULES psoriasis, urticaria; Rare: erythema nodosum, exfoliative dermatitis, lichenoid dermatitis, hair discoloration, skin discoloration, furunculosis, hirsutism, leukoderma, petechial rash, pustular rash, vesiculobullous rash, seborrtea, skin atrophy, skin striae. Special senses - Frequent: abnormality of accommodation, mydriasis, taste perversion; Infrequent: cataract, conjunctivitis, comeal lesion, diplopia, dry eyes, eye pain, conjunctival edema, deafness, exophthalmos, glaucoma, retin hemorhthage, subconiunctival hemornage, keratitis, labyrinthitis, miosis, papilledema, decreased pupillary reflex, otitis externa, scleritis, uveitis. Urogenital system - Frequent: dysuria, metrorrhagia, "prostatic disorder (prostatitis and enlarged prostate)," unination impaired, vaginitis"; Intrequent albuminunia, amenontea," cystitis, hemauria, leukorntea,

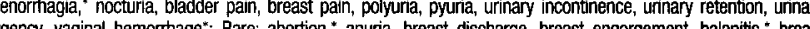
urgency, vaginal hemormage*; Rare: abortion," anuria, breast discharge, breast engorgement, balanitis," breast

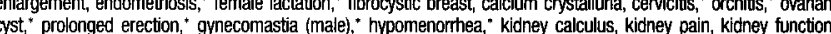
cyst," prolonged erection," gynecomasta (male)," hypomenormhea," kidney calculus, kidney pain, kidney function
abnormal, mastitis, menopause," pyelonephritis, oliguria, salpingitis," urolithiasis, uterine hemornthage," uterine spasm." ("Based on the number of men and women as appropriate). Postmarketing Reports: agranulocytosis, anaphylaxis, aplastic anemia, catatonia, congenital anomalies, CPK increased, deep vein thrombophlebitis, delirium, EKG abnormalities such as QT prolongation; cardiac arrtythmias including atrial fibrillation, supraventricular tachycardia, exidermal necrosis/Stevens-Johnson Syndrome, erythema multiforme, extrapyramidal symptoms (including tardive dyskinesia), hemornhage (including eye and gastrointestinal bleeding), hepatic events (including GGT elevation; abnormalities of unspecified liver function tests; liver damage, necrosis, or failure; and fatty liver), involuntary movements, LDH increased, neuroleptic malignant syndromoered), neutropenia night se of a 10-year-old who may have panic, prolactin increased, pulmonary eosinophilia, renal failure, serotonin syndrome, shock-Ilke electrical sensations (in some cases, subsequent to the discontinuation of venlafaxine or tapering of dose), and syndrome of inappropriate antidiuretic hormone secretion (usually in the elderlyh There have temporally associated with adverse events, including seizures, following the addition of venlafaxine. There have been eports of increases in prothrombin time, partial thromboplastin time, or INR when venlafaxine was given to patients receiving warfarin therapy. DRUG ABUSE AND DEPENDENCE: Effexor XR is not a controlled substance. Evaluat patients carefully for history of drug abuse and observe such patients closely for signs of misuse or abuse. OVERDOSAGE: Electrocardiogram changes (e.g., prolongation of $Q T$ interval, bundle branch block, ORS prolongation), sinus and ventricular tachycardia, bradycardia, hypotension, altered level of consciousness (ranging from somnolence to coma), seizures, vertigo, and death have been reported. Treatment should consist of those general measures employed in the management of overdosage with any antidepressant. Ensure an adequate airway, oxygenation and mented. Induction of emesis is not recommended. Gastric lavage with a large bore orogastric tube with appropriate aiway protection, if needed, may be indicated if performed soon after ingestion or in symptomatic patients. Activated charcoal should be administered. Due to the large volume of distribution of this drug, forced diuresis, dialysis, hemoperusion, and exchange transtusion are unlikely to be of benefit No specific antidotes for venlafaxine are known. In managing overdosage, consider the possibility of multiple drug involvement. The physician should consider contacting poison control center for additional information on the treatment of any overdose. Telephone numbers for certified poison control centers are listed in the Physicians' Desk Reference* (PDR) DOSAGE AND ADMinISTRATION. Please consult full prescribing information for detailed dosing instructions. Discontinuing Effexor $X \mathrm{R}-$ When discontinuing Effexor XR, the dose should be tapered gradually, based upon the dose, duration of therapy and the individual patien. Discontinuation symptoms reported include agitation, anorexia, anxiety, contusion, coordination impaired, diarthea, dizziness, dry moutth, dysphoric mood, fasciculation, fatigue, headaches, hypomania, insomnia, nausea, nervousness, nightmares, sensory disturbances (including shock-like electrical sensations), somnolence, sweating, temor, vertigo and vomiting. Switching Patients To or From a Monoamine Oxidase Inhibitor-At least 14 days should elapse allowed after stopping Effexor XR before starting an MAOI (see "Contraindications" and "Warnings"). This brief summary is besed on the circular $\mathrm{Cl} 7509-4$, revised April 11, 2002. 


\section{Something}

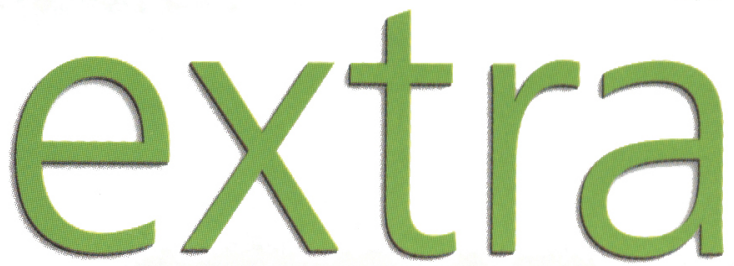

... approximately

$1 / 3$ more

patients got their life back

In a pooled analysis of over 2,000 patients, against leading SSRIs (fluoxetine, paroxetine, fluvoxamine),

EFFEXOR XR/EFFEXOR offered something extrain depression, remission* of symptoms in approximately

$1 / 3$ more patients. ${ }^{\prime}$

EFFEXOR XR is contraindicated in patients taking monoamine oxidase inhibitors (MAOIs). EFFEXOR XR should not be used in combination with an MAOI or within at least 14 days of discontinuing treatment with an MAOI; at least 7 days should be allowed after stopping EFFEXOR XR before starting an MAOI. The most common adverse events reported in EFFEXOR XR placebocontrolled depression trials (incidence $\geq 10 \%$ and $\geq 2 x$ that of placebo) were nausea, dizziness, somnolence, delayed ejaculation, sweating, dry mouth, and nervousness; and in GAD trials were nausea, dry mouth, insomnia, delayed ejaculation, anorexia, constipation, nevousness, and sweating. Treatment with venlafaxine is associated with sustained increases in blood pressure (BP) in some patients. Three percent of EFFEXOR XR patients in depression studies (doses of 75 to $375 \mathrm{mg} /$ day) and $0.5 \%$ in GAD studies (doses of 37.5 to $225 \mathrm{mg} /$ day) had sustained $\mathrm{BP}$ elevations. Less than $1 \%$ discontinued treatment because of elevated BP. Regular BP monitoring is recommended.

Patients should not be abruptly discontinued from antidepressant medication, including EFFEXOR XR. See the Dosage and Administration section of the Prescribing Information. References: 1. Thase ME, Entsuah AR, Rudolph RL. Remission rates during treatment with venlafaxine or selective serotonin reuptake inhibitors. Br J Psychiatry. 2001;178:234-241. 2. Kupfer DJ. Long-term treatment of depression. I Clin Psychiatry. 1991;52(5, suppl):28-34. Please see brief summary of Prescribing Information on adiacent page.

Visit us at www.EFFEXORXR.com

Remission of symptoms

is a first step on the

road to recovery. ${ }^{2}$

* Remission is defined as minimal or no symptoms (HAM-D $\leq 7$ ). ${ }^{1}$

Indicated in Depression and

Generalized Anxiety Disorder

ONCE-DAILY

VENLAFAXINE HCl GFFEXOR XR 